1 Taking the next-gen step: comprehensive antimicrobial resistance detection from Burkholderia

\title{
pseudomallei genomes
}

3

4 Danielle E. Madden BSc (Hons) ${ }^{1,2}$, Jessica R. Webb $\mathrm{PhD}^{3}$, Eike J. Steinig BSc (Hons) ${ }^{4}$, Bart J. Currie 5 FRACP ${ }^{3,5}$, Erin P. Price $\mathrm{PhD}^{1,2,3^{\wedge}}$, and Derek S. Sarovich $\mathrm{PhD}^{1,2,3^{\wedge} \#}$

6

$7{ }^{1}$ GeneCology Research Centre, University of the Sunshine Coast, Sippy Downs, Queensland,

8 Australia

$9 \quad{ }^{2}$ Sunshine Coast Health Institute, Sunshine Coast University Hospital, Birtinya, Queensland, Australia

$10{ }^{3}$ Global and Tropical Health Division, Menzies School of Health Research, Charles Darwin University,

11 Tiwi, Northern Territory, Australia

$12{ }^{4}$ Australian Institute of Tropical and Health Medicine, James Cook University, Townsville, Queensland,

13 Australia

$14{ }^{5}$ Department of Infectious Diseases and Northern Territory Medical Program, Royal Darwin Hospital,

15 Tiwi, Northern Territory, Australia

16

17 Keywords: ARDaP, antimicrobial resistance, comparative genomics, next-generation sequencing,

18 bioinformatics

19

20 Running title: Antibiotic resistance detection from B. pseudomallei genomes

21

$22 \wedge$ Authors contributed equally

23 \#Corresponding author

24 Derek S. Sarovich

25 dsarovich@usc.edu.au

$26+61754563748$

27 Locked Bag 4, Maroochydore DC, Queensland, 4558, Australia 


\section{Abstract}

29 Background: Antimicrobial resistance (AMR) poses a major threat to human health. Whole-genome

30 sequencing holds great potential for AMR identification; however, there remain major gaps in

31 comprehensively detecting AMR across the spectrum of AMR-conferring determinants and pathogens.

Methods: Using 16 wild-type Burkholderia pseudomallei and 25 with acquired AMR, we first assessed

33 the performance of existing AMR software (ARIBA and CARD) for detecting clinically relevant AMR in

34 this pathogen. B. pseudomallei was chosen due to limited treatment options, high fatality rate, and

35 AMR caused exclusively by chromosomal mutation (i.e. single-nucleotide polymorphisms [SNPs],

36 insertions-deletions [indels], copy-number variations [CNVs], and functional gene loss). Due to poor

37 performance with existing tools, we developed ARDaP (ㅁntimicrobial Resistance Detection and

38 Prediction) to identify the spectrum of AMR-conferring determinants in B. pseudomallei.

39 Results: CARD failed to identify any clinically-relevant AMR in B. pseudomallei, ARIBA cannot

40 differentiate AMR determinants from natural genetic variation, and neither CARD or ARIBA can

41 identify CNV or gene loss determinants. In contrast, ARDaP accurately detected all SNP, indel, CNV,

42 and gene loss AMR determinants described in B. pseudomallei ( $n \approx 50)$. Additionally, ARDaP

43 accurately predicted three previously undescribed determinants. In mixed strain data, ARDaP

44 identified $\mathrm{AMR}$ to as low as $\sim 5 \%$ allelic frequency.

45 Conclusions: We demonstrate that existing AMR software are inadequate for comprehensive AMR

46 detection; ARDaP overcomes the shortcomings of existing tools. Further, ARDaP enables AMR

47 prediction from mixed sequence data down to $5 \%$ allelic frequency. ARDaP databases can be

48 constructed for any microbial species of interest for comprehensive AMR detection. 


\section{Introduction}

Antimicrobial resistance (AMR) poses a major threat to human health worldwide and an increasing contributor to morbidity and mortality. Antibiotic use and misuse have resulted in an alarming increase in multidrug-resistant infections worldwide, resulting in an urgent need to improve global AMR detection and surveillance. Alongside pathogen identification, AMR detection is one of the primary goals of diagnostic microbiology, with far-reaching consequences for both infection control and effective treatment [1].

Whole-genome sequencing (WGS) holds great potential for comprehensive AMR detection and prediction from bacterial genomes by identifying all AMR determinants in a single genome or metagenome [2], circumventing the need for multiple and often laborious diagnostic methods. Existing bioinformatic tools such as ARG-ANNOT [3], Antibiotic Resistance Identification By Assembly (ARIBA) [4], Comprehensive Antibiotic Resistance Database (CARD) [5], and MEGARes [2] can readily detect AMR genes acquired from horizontal gene transfer events. Many bacterial pathogens also develop AMR via chromosomal mutations, including missense single-nucleotide polymorphism (SNP) mutations in $\beta$-lactamase-encoding genes, SNPs or nonsense insertion-deletions (indels) in efflux pump regulators [6-8], gene amplification via copy-number variations (CNVs) [9], and functional gene loss [6]. Recent improvements in AMR identification software mean that chromosomal mutations, particularly SNPs, are now identifiable. For example, ARIBA can identify AMR-conferring SNPs and indels in multiple species [4]. Nevertheless, other types of genetic variants -particularly gene loss or truncation, inversions, and CNVs - remain poorly identified using existing tools, despite their crucial role in conferring AMR [10].

The bacterium, Burkholderia pseudomallei, causes the often-fatal tropical disease melioidosis. Melioidosis severity ranges from mild, self-limiting skin abscesses to pneumonia, neurological disease, and septic shock. B. pseudomallei is naturally resistant to many antibiotics, including aminoglycosides, penicillins, macrolides, and polymyxins [11, 12]. Fortunately, human-to-human $B$. pseudomallei transmission is rare; almost all infections are acquired from the environment. As such, isolates collected prior to antibiotic treatment are almost universally susceptible to the following clinically-relevant antibiotics: ceftazidime (CAZ), amoxicillin-clavulanate (AMC), co-trimoxazole (SXT), doxycycline (DOX), meropenem (MEM) and imipenem (IPM) [13]. To prevent melioidosis relapse, 
78 treatment involves prolonged (3-6 month) antibiotic therapy, which increases AMR risk and treatment

79 failure [6]. AMR in B. pseudomallei has been reported for all clinically-relevant antibiotics [6], with

80 novel AMR determinants towards these key antibiotics continuing to be uncovered.

81 Here, we tested 47 characterised B. pseudomallei genomes with known antibiotic phenotype profiles

82 and associated AMR determinants, and three MEM-resistant (MEMr) strains with previously

83 unidentified AMR determinants, against existing tools (ARIBA and CARD) to determine their AMR

84 detection efficacy. Among the characterised strains, 25 were phenotypically-confirmed as resistant

85 towards at least one clinically relevant antibiotic, 16 were sensitive, and the remainder encoded

86 unusual sensitivity towards aminoglycosides and macrolides or stepwise AMR variants. Following

87 testing against the current AMR tools, we developed a new tool, Antibiotic Resistance Detection and

88 Prediction (ARDaP), to permit comprehensive AMR detection from microbial genomes. ARDaP was

89 designed to meet four main aims: first, to accurately identify AMR determinants caused by a spectrum

90 of mutational mechanisms (i.e. gene gain, SNPs, indels, CNVs, and functional gene loss); second, to

91 predict enigmatic AMR determinants in isolates with phenotypically-confirmed AMR, third, to detect

92 minor AMR allelic determinants in mixed (e.g. metagenomic) sequence data; and finally, to provide a

93 user-friendly report that summarises the AMR determinants (if any) and associated AMR phenotypes,

94 stepwise variants, unusual antimicrobial sensitivity determinants, and genetic variants associated with

95 natural variation that do not confer AMR. Although we illustrate its utility in $B$. pseudomallei, ARDaP is

96 amenable to AMR identification across all microbial species. 


\section{Methods}

Ethics. Ethical approval was obtained as previously described [14].

Isolates. Forty-seven B. pseudomallei strains were included in this study, including 25 with elevated MICs towards one or more clinically-relevant antibiotics (Table 1) and genotypically-confirmed AMR determinants. These isolates were selected as they represent the spectrum of known AMR determinants in B. pseudomallei (Table S1). Strains encoding unusual aminoglycoside- and macrolide-sensitivity, and stepwise mutations that lower the barrier to AMR development, were also examined (Table 1). A further 16 strains sensitive to all clinically-relevant antibiotics were included to test software efficacy (Table 2). Finally, three previously uncharacterised clinical strains exhibiting

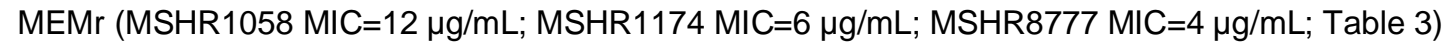
were included to test the predictive capacity of ARDaP.

Culturing, WGS, and genome assembly. B. pseudomallei culture, DNA extraction, and WGS were performed as described elsewhere [9]. Genomic data for MSHR1058, MSHR1174, and MSHR8777 were uploaded to the NCBI Sequence Read Archive database (BioProject PRJNA641249). Accession numbers for all other genomic data are listed in Tables 1 and 2. For genomes lacking a publiclyavailable assembly, MGAP v1.1 (https://github.com/dsarov/MGAP---Microbial-Genome-AssemblerPipeline) was used, with archetypal strain K96243 (RefSeq accessions NC_006350.1 and NC_006351.1) provided as the scaffolding reference.

Minimum Inhibitory Concentrations (MICs). MICs were determined using Etests (bioMérieux, Murarrie, Australia). Sensitive, intermediate and resistant cut-offs were based on the Clinical and Laboratory Standards Institute (CLSI) M100-S17 guidelines for $B$. pseudomallei $(\leq 8 / 4,16 / 8$, and $\geq 32 / 16 \mu \mathrm{g} / \mathrm{mL}$ for AMC; $\leq 8,16, \geq 32 \mu \mathrm{g} / \mathrm{mL}$ for CAZ; $\leq 4,8, \geq 16 \mu \mathrm{g} / \mathrm{mL}$ for DOX and IPM; $\leq 2 / 38$, nil, $\geq 4 / 76 \mu \mathrm{g} / \mathrm{mL}$ for SXT). CLSI guidelines do not list MEM for B. pseudomallei; however, based on prior work [15, 16], and recent proposed EUCAST breakpoints for B. pseudomallei, we categorised MEMr as $\geq 3 \mu \mathrm{g} / \mathrm{mL}$. Likewise, the CLSI guidelines do not list gentamicin (GEN) MIC values for $B$. pseudomallei due to almost ubiquitous resistance $(>16 \mu \mathrm{g} / \mathrm{mL})$ towards this antibiotic; however, there are notable exceptions [14, 17]. We chose a GEN-sensitive cut-off of $\leq 4 \mu \mathrm{g} / \mathrm{mL}$, which also reflects those strains unable to grow on Ashdown's agar, a selective medium for B. pseudomallei isolation that contains $4 \mu \mathrm{g} / \mathrm{mL}$ GEN. 
126 AMR software parameters. The default RGI v5.1.0 database parameters of CARD v3.0.9

127 (https://card.mcmaster.ca/analyze/rgi; accessed 25Jun20), and ARIBA v2.14.5

128 (https://github.com/sanger-pathogens/ariba), were examined for performance across the $B$.

129 pseudomalleigenomes.

130 ARDaP AMR database construction. ARDaP is available at: https://github.com/dsarov/ARDaP. All

131 reported B. pseudomallei AMR determinants, including stepwise AMR mutations and unusual

132 antimicrobial susceptibility mutations (Table 1; Table S1), were annotated relative to K96243. The

133 AMR determinants (as of version 1.7) are summarised in an SQLite database (Table S1; most up-to-

134 date version available at:

135 https://github.com/dsarov/ARDaP/tree/master/Databases/Burkholderia pseudomallei k96243).

136 Briefly, CAZ resistance (CAZr) is caused by altered PenA $\beta$-lactamase substrate specificity [18-21],

137 penA upregulation [7, 20, 22, 23] (including CNVs [9]), or loss of penicillin-binding protein 3 [24]; AMC

138 resistance (AMCr) is caused by penA upregulation [14, 20]; MEMr is caused by AmrAB-OprA, BpeAB-

139 OprB, or BpeEF-OprC resistance-nodulation-division (RND) multidrug efflux pump regulator loss-of-

140 function [6]; SXT resistance (SXTr) is caused by cumulative mutations in core metabolism pathways

141 coupled with AmrAB-OprA, BpeAB-OprB, or BpeEF-OprC RND efflux pump regulator loss-of-function

$142[6,9,25]$; and DOX resistance (DOXr) is caused by loss-of-function mutations within the SAM-

143 dependent methyltransferase gene, BPSL3085, often in combination with AmrAB-OprA, BpeAB-OprB,

144 or BpeEF-OprC regulator loss-of-function [26]. Our B. pseudomallei ARDaP database also includes

145 AmrA and AmrB mutants that are associated with unusual aminoglycoside and macrolide

146 susceptibility $[14,17]$. To avoid poor-quality WGS data or incorrect species assignments, the database

147 also includes two conserved genetic targets (Table S1) found only in this bacterium; strains lacking

148 these loci are flagged for further user assessment.

149 ARDaP algorithm. To achieve high-quality variant calls, ARDaP incorporates several tools into its

150 workflow (full list available at: https://github.com/dsarov/ARDaP). In addition to an organism-specific

151 SQLite database (https://github.com/dsarov/ARDaP/tree/master/Databases), ARDaP requires WGS

152 data, either genomes or metagenomes in paired-end Illumina v1.8+ FASTQ format, or assembled

153 genomes in FASTA format, as input (Figure 1). For genomes in FASTA format, ARDaP first converts

154 to synthetic Illumina v1.8+ reads. For genomes in FASTQ format, ARDaP performs quality filtering 
155 followed by optional random down-sampling to a user-defined coverage (default=50x) to permit more

156 rapid analysis. ARDaP then performs comparative genomic analysis to identify AMR determinants by

157 mapping reads against an annotated reference, followed by alignment processing, SNP and indel

158 identification, coverage assessment, and structural variant identification. High-quality genetic variants

159 (SNPs, indels [<50bp], CNVs, gene gain, and gene loss or truncation) are then annotated. ARDaP

160 next interrogates two databases: i) a customisable CARD [5] database is screened to identify

161 horizontally-acquired AMR genes and to ignore conserved genes that do not confer AMR, and ii) a

162 bespoke AMR determinant database (in this study, a B. pseudomallei database) containing species

163 specific AMR determinants. ARDaP databases are created in SQLite and can be readily updated as

164 additional AMR determinants are identified. This database also accommodates stepwise mutations

165 and AMR conferred by $\geq 2$ mutations. Finally, ARDaP can predict AMR by identifying novel high-

166 consequence mutations (i.e. those resulting in a frameshift or nonsense mutations) in known AMR

167 genes. These putative mutants can then be flagged for further investigation with phenotypic AMR

168 testing. ARDaP outputs are presented in a comprehensive, human-readable report (Figure 3), which

169 has been modelled from the report structure described by Crisan and colleagues .

170 Mixture detection. ARDaP incorporates a minor allelic variant analysis function to permit variant

171 identification from mixed genomes/metagenomes, enabling the detection of emerging AMR

172 determinants (down to 5\% abundance). Minor-variant SNPs and indels are identified using the ploidy-

173 aware HaplotypeCaller tool in GATK v4.1; deletions, CNVs, and structural mutations are identified with

174 the ploidy-aware function of Pindel. B. pseudomallei strains with known AMR status were mixed at

175 ratios of $5 \%$ increments ranging from 5:95 to $95: 5$, to $55-60 x$ total depth. Two mixtures were created:

176 MSHR0913 (sensitive to all clinically-relevant antibiotics) and MSHR0937 (MEMr; intermediate

177 resistance to AMC and SXT), and MSHR0913 and MSHR8441 (SXTr; decreased susceptibility to

178 CAZ, MEM, and DOX). MSHR0937 and MSHR8441 were chosen as they represent a wide spectrum

179 of clinically-relevant AMR and mutation types (Table 4). 


\section{Results and Discussion}

181 Performance of existing AMR tools in B. pseudomallei. The validated dataset of $47 \mathrm{~B}$.

182 pseudomallei isolates was used to assess the performance and capacity of existing AMR tools to 183 identify AMR determinants in AMR but not antimicrobial-sensitive strains. According to CARD, all 47 184 genomes were found to harbour AMR determinants; however, all determinants corresponded with 185 conserved genes in B. pseudomallei (Table S2). In addition, CARD failed to identify any clinically 186 relevant AMR determinants in the 25 AMR strains. This finding is consistent with B. pseudomallei 187 acquiring AMR through chromosomal mutation rather than gene gain, and an inability of current CARD 188 software to identify AMR variants conferred by indels, CNVs, or gene loss/truncation. ARIBA 189 outperformed CARD due to its ability to include missense (although not nonsense) SNPs in its 190 database construction and to identify SNPs and indels in its report outputs. However, we encountered 191 several shortcomings with ARIBA; notably, an inability to differentiate AMR determinants from natural 192 variation, cumbersome and labour-intensive input file requirements, restrictions on database 193 construction (e.g. reference genomes with indels and nonsense mutations cannot be included), and 194 an inability to identify CNVs. In our hands, ARIBA provided comparable information to variant 195 reporting outputs generated by comparative genomic pipelines. However, such efforts require 196 extensive domain-specific knowledge to accurately uncover and interpret AMR determinants. In 197 contrast, ARDaP uses a single and standardised SnpEff-based input, can differentiate natural gene 198 variation from known and putative AMR determinants, can detect CNVs, and provides a user-friendly 199 output that does not require domain-specific knowledge to interpret outputs.

200 ARDaP development and performance in B. pseudomallei. Due to shortcomings in existing AMR 201 software, ARDaP was designed to both identify known AMR determinants and to ignore non-causal 202 genetic variants (Table 2). ARDaP correctly identified all B. pseudomallei AMR determinants (Table 203 1); however, when tested against the 47 validated isolates, one false negative and four false positives 204 were identified. The false negative result (SXT sensitivity rather than decreased SXT susceptibility) 205 was due to an $800 \mathrm{~kb}$ inversion in 354e that directly impacts $\| \mathrm{pE}$, a gene that resides between bpeT 206 and bpeEF-oprC [7]. BpeT is a LysR-type transcriptional regulator that controls expression of BpeEF207 OprC [27]. This inversion was not detected due to limitations in short-read Illumina data that led to this 
208 structural variant not easily being identified by Pindel, coupled with the occurrence of this inversion 209 outside of an AMR determinant.

210 The first of the false positives, chronic cystic fibrosis (CF) isolate MSHR5654, was predicted to be

211 MEMr due to the presence of BpeT Thr314fs. However, Etesting showed MEM sensitivity $(2 \mu \mathrm{g} / \mathrm{mL})$ in this

212 strain, just below the MEMr threshold ( $\geq 3 \mu \mathrm{g} / \mathrm{mL}$ ) [9, 23]. Although alterations in BpeT have been

213 putatively linked with MEMr in MSHR1300 (4 $\mu \mathrm{g} / \mathrm{mL})$ [6] and 354e $(6 \mu \mathrm{g} / \mathrm{mL})$ [7], the role of BpeT

214 mutations in conferring MEMr is contentious [25]. In support of this notion, MSHR1300 also encodes

215 AmrR $_{\mathrm{K} 13 \mathrm{fs}}$, a TetR-family cis-acting repressor of the AmrAB-OprA RND efflux pump, which likely

216 causes MEMr in its own right [6], and in 354e, the $~ 800 \mathrm{~kb}$ inversion likely also affects other AMR-

217 conferring genes besides bpeT. As such, the B. pseudomallei ARDaP database was updated to flag

218 bpe $T$ variants as stepwise mutations rather than solely conferring MEMr (Table 1; Table 2), thereby

219 correcting the original false-positive call for MSHR5654. This issue highlights the complexity of

220 unravelling AMR determinants, and in this case, the need for additional work to determine a role, if

221 any, for bpeT mutations in conferring MEMr.

222 The remaining false-positive strains, MSHR5666, MSHR5669, and MSHR5670, were all obtained from 223 a chronically-infected CF patient, CF9 [9]. All encode a SAM-dependent methyltransferase truncation 224 (BPSL3085 ${ }_{\text {A88fs }}$ ) [23] and were predicted by ARDaP to be DOXr. We also observed BPSL3085 ${ }_{A 88 f s}$ in 225 an unrelated DOXr chronic CF strain, Bp1651 [28] (Table 1). BPSL3085 mutations confer DOXr by 226 altering ribosomal methylation patterns $[9,26]$. However, all three strains remained DOX-sensitive $(1.5$ $227 \mu \mathrm{g} / \mathrm{mL}$ ) despite other CF9 strains encoding BPSL3085 ${ }_{\text {A88fs }}$ and being DOXr (MSHR5665: MIC=6 $228 \mu \mathrm{g} / \mathrm{mL}$; MSHR5667: MIC=48 $\mu \mathrm{g} / \mathrm{mL}$; Table 1) [9]. The higher DOX MIC in MSHR5667 is attributable to 229 a second mutation (AmrR L132P $_{1}$ Table 1). We postulate that MSHR5666, MSHR5669, and MSHR5670 230 encode an unidentified mutation that reverts them to a DOX-sensitive phenotype. Notably, all 231 longitudinal CF9 isolates, including MSHR5666 and MSHR5669, encode mutS mutations, resulting in 232 a hypermutator phenotype [9, 23]. Therefore, identifying the causal basis for this reversion is non233 trivial due to the large number of mutations (range: 112-157) accrued by these hypermutator strains 234 [9]. In addition, MSHR5670 was predicted to be SXTr due to Ptr1 $1_{\mathrm{W}_{116 R}}$, MetF $\mathrm{Q}_{\mathrm{Q} 28 \mathrm{P}}$, and Dut $\mathrm{V}_{\mathrm{V} 7 \mathrm{~A}}$ variants, 235 yet exhibited SXT sensitivity (Table 2). The cause of SXTr reversion in this strain is also currently 236 unknown. 
237 Importance of including natural genetic variation in AMR databases. Accurate prediction of novel

238 AMR determinants requires thorough cataloguing of both confirmed AMR-causing mutations and

239 natural variation in AMR-encoding genes to avoid false positives. As an illustration, a PenA $\beta$ -

240 lactamase missense mutation (K96243 numbering: PenA S78F $_{3}$; encoded by BPSS0946) has been

241 linked to $\mathrm{AMCr}[18,21,28]$. However, we found that $\mathrm{PenA}_{\mathrm{S} 78 \mathrm{~F}}$ alone is unlikely to cause $\mathrm{AMCr}$ due to

242 its presence in genetically diverse AMC-sensitive strains ( $M I C=3-4 \mu \mathrm{g} / \mathrm{mL}$ in strains MSHR0291,

243 MSHR0668, MSHR0848, MSHR0911, MSHR1711, MSHR2212, MSHR3902, MSHR4797,

244 MSHR8392, and MSHR9887). Instead, AMCr is likely conferred by both PenA $\mathrm{A}_{\mathrm{S78F}}$ and penA

245 upregulation, the latter of which can be caused by mutations within the 5' untranslated region [20] or

246 penA CNVs [9]. We therefore included $\mathrm{PenA}_{\mathrm{S} 78 \mathrm{~F}}$ as a putative stepwise mutation (Table 2), with an

247 additional penA upregulation mutation required to confer the AMCr phenotype. In another example,

248 we observed that both AMR and antimicrobial-sensitive strains can possess 3'-truncated amrR (Table

249 2). Multiple frameshift mutations and deletions in amrR are associated with MEMr [6] due to loss-of-

250 repressor function (Figure 3). However, 3' region mutations (residues 210-223) do not cause MEMr

251 (Table 2). To accommodate this natural genetic variation, we coded ARDaP to ignore these non-

252 causal 3' variants, thereby greatly reducing false-positive MEMr rates.

253 No evidence of IPM-resistant B. pseudomallei. In most melioidosis treatment guidelines, IPM has

254 been replaced by MEM due to neurotoxicity concerns.[29] However, the recent discovery of MEMr $B$.

255 pseudomallei has resurrected IPM as a treatment option due to a lack of cross-resistance between

256 these carbapenems [6] and exceedingly low rates of reported IPM resistance (IPMr) [30]. The one

257 study reporting an IPMr (MIC=8 $\mu \mathrm{g} / \mathrm{mL})$ B. pseudomallei strain, Bp1651, attributed this phenotype to a

$258 P^{P e n A_{T 147 A}}$ mutation (K96243 numbering: PenA $\mathrm{T}_{T 153 \mathrm{~A}}$ ) combined with upregulation due to a promoter

259 mutation [28]. We subsequently refuted the role of the $\mathrm{PenA}_{\mathrm{T} 147 \mathrm{~A}}$ variant alone in conferring IPMr by

260 identifying three genetically unrelated PenA ${ }_{T 153 \mathrm{~A}}$-encoding strains that were IPM-sensitive [6]. Further,

261 this variant is dominant (>50\%) in publicly available B. pseudomallei genomes, most of which exhibit

262 wild-type antimicrobial sensitivity. Given that $P_{e n A_{T 147 A}}$ occurs at a very high rate in the wild-type $B$.

263 pseudomallei population, and none have been shown to exhibit IPMr, this mutant has not been

264 included in our ARDaP database. However, this variant can readily be added as a stepwise AMR

265 determinant should further evidence come to light about its role in conferring AMR. 
266 Reversions and unusual antimicrobial susceptibility. Aminoglycoside- and macrolide-class

267 antibiotics are typically not included in melioidosis treatment regimens due to near-ubiquitous intrinsic

268 resistance; indeed, GEN resistance is commonly used for B. pseudomallei selection [17]. However,

269 rare cases of sensitivity have been documented, such as in ST-881 and ST-997 strains from Sarawak,

270 Malaysian Borneo, which naturally encode AmrB $_{\text {T368R }}$, resulting in AmrAB-OprA loss-of-function and

271 unusual aminoglycoside and macrolide susceptibility [17]. An AmrAB-OprA loss-of-function variant has

272 also been described in Bp1651 ( $\left.\mathrm{AmrB}_{\mathrm{A} 254 \mathrm{ss}}\right)$ [28]. Although ARDaP detected amrR loss in MSHR1043,

273 the co-presence of $\operatorname{amrA}$ loss $\left(\mathrm{AmrA}_{\mathrm{L} 247 \mathrm{fs}}\right)$ resulted in reversion of MEMr to a wild-type MIC $(0.75$

$274 \mu \mathrm{g} / \mathrm{mL}$ ). This reversion also causes unusual gentamicin ( $\mathrm{MIC}=1 \mu \mathrm{g} / \mathrm{mL})$ [14] and presumably

275 kanamycin and azithromycin sensitivity. Given their confounding potential, we incorporated these

276 reversions into ARDaP to more accurately reflect the true strain phenotype. In addition, strains

277 encoding AmrAB-OprA loss-of-function variants are at far lower risk of developing MEMr than wild-

278 type strains, supporting longer-term MEM use in such cases. These findings highlight the value of

279 including sensitivity-conferring variants in AMR prediction databases by increasing the antibiotic

280 arsenal in naturally multidrug-resistant pathogens where treatment options are limited.

281 AMR predictive capacity of ARDaP. We tested ARDaP's predictive capacity to identify the causative 282 mutation/s in three clinical MEMr strains (MSHR1058, MSHR1174, and MSHR8777; MEM MIC range:

$2834-12 \mu \mathrm{g} / \mathrm{mL}$; Table 3) with no previously reported AMR determinants. ARDaP identified novel amrR

284 mutations in each strain, all of which resulted in AmrR loss-of-function $\left(\mathrm{AmrR}_{\triangle \mathrm{P} 81-\mathrm{H} 223}\right.$ in MSHR1058;

285 AmrR $_{\mathrm{G} 149 \mathrm{fs}}$ in MSHR1174; $\mathrm{AmrR}_{\triangle \mathrm{A} 128-\mathrm{H} 223}$ in MSHR8777; Figure 3). All patients received MEM

286 treatment prior to isolate retrieval, consistent with selection pressure driving AMR. These findings

287 provide further confirmation of the link between MEM treatment and potential treatment failure due to

288 AmrR mutability [6], and demonstrate the value of ARDaP for predicting novel AMR determinants.

289 ARDaP performance on mixed sequence data. The ARDaP algorithm is mixture-aware, an

290 important feature for detecting emerging AMR determinants in mixed strain data (e.g. non-purified

291 colonies, culture sweeps, clinical specimens). To assess the performance of the mixture function in

292 ARDaP, Illumina reads from antimicrobial-sensitive and AMR B. pseudomallei strains (Table 4) were

293 mixed at 5\% incremental ratios ranging from 5:95 to 95:5. ARDaP identified one AMR determinant

294 down to the lowest tested ratio of 5\% minor allele frequency: a penA 10x CNV from MSHR8441 (Table 
295 4). The other determinants were identified by ARDaP when present at minor allele frequencies of $10 \%$

296 (BPSL3085 1 S130L $), 20 \%\left(\right.$ BpeR $\left._{\text {Asp176Ala }}\right), 25 \%\left(\right.$ Ptr1 $_{\text {A22_G23ins_R-R-A }}$ and $\left.\operatorname{MetF}_{\text {Gin } 142^{*}}\right)$ and $30 \%\left(\operatorname{AmrR}_{\Delta V 62-}\right.$

297 н223) (Table 4). Although CNVs were readily identified at low allelic frequencies, and the detection of

298 SNPs and indels ranged from 10 to $25 \%$, gene truncations had the lowest sensitivity due to the

299 challenge of discriminating gene loss from Illumina depth coverage variation, coupled with inherent

300 limitations in short-read data mapping. Next, ARDaP was tested on a previously detected AmrR

301 mixture from strain MSHR9021, which encodes $A_{m r R} R_{166 P}$ and $A_{m r R} R_{A 145 f s}$ variants at $~ 66 \%$ and $~ 33 \%$

302 allele frequencies, respectively ${ }^{6}$ (Table 1). ARDaP detected $\mathrm{AmrR}_{\mathrm{S} 166 \mathrm{P}}$ and $\mathrm{AmrR}_{\mathrm{A} 145 \text { ss }}$ at allele

303 frequencies of $63 \%$ and $31 \%$, respectively, thus closely reflecting their known proportions. Overall,

304 ARDaP confidently identified all mixtures, albeit with varying sensitivities. Further validation on specific

305 variant mixtures is recommended when new mixtures are identified to determine their sensitivity.

306 Deeper sequencing (e.g. 100-500x) should enable more robust mixture detection at lower allele

307 frequencies.

308 ARDaP reports. ARDaP generates an easy-to-interpret report that summarises the AMR

309 determinants and associated antibiotic phenotype/s for each genome. This report summarises AMR

310 findings for first-line, second-line, and tertiary antibiotics (Figure 2), along with instances of unusual

311 antibiotic susceptibility, and has been designed to prioritise a clinical workflow. This report also lists

312 stepwise AMR determinants, thereby informing early treatment shifts that mitigate the risk of AMR

313 emergence and fixation. This easy-to-interpret report represents a major improvement over current

314 software for AMR annotation such as ARIBA and CARD, both of which require an intimate

315 understanding of AMR determinants to correctly interpret outputs and to ignore natural genetic

316 variation. The AMR report produced by ARDaP represents an important step towards the

317 incorporation of WGS as a routine tool for guiding best-practice AMR stewardship and personalised

318 treatment regimens in the clinical diagnostic setting. 


\section{Funding}

320 This work was supported by the National Health and Medical Research Council (1046812 and

3211098337 to B.J.C., E.P.P., and D.S.S.; and 1131932 to B.J.C.), Advance Queensland

322 (AQIRF0362018 to E.P.P, and AQRF13016-17RD2 to D.S.S.), the Australian Government (Research

323 Training Scholarship to D.E.M.), and James Cook University (International Postgraduate Research

324 Scholarship to E.S.). The funders had no role in study design; in the collection, analysis, or

325 interpretation of data; in the writing of this report; or in the decision to submit for publication. The

326 corresponding author had full access to all the data in the study and has final responsibility for the

327 decision to submit for publication.

\section{Acknowledgements}

329 We thank Associate Professor Rob Baird and the microbiology staff at Royal Darwin Hospital for their 330 support and expertise in identifying and characterising B. pseudomallei isolates, and Vanessa Rigas,

331 Glenda Harrington, and Mark Mayo for isolate inventory support.

\section{Author contributions}

333 DSS conceived of the study; EPP and DSS designed the study; JRW, EPP, and DSS generated

334 laboratory data and performed laboratory analyses; EJS and DSS wrote the software; DEM, EPP, and

335 DSS performed data analysis, literature searches, figure generation, software testing, and feature

336 development; BJC provided isolates; DEM, EPP, and DSS wrote the manuscript; and BJC, EPP, and

337 DSS obtained funding for the study. All authors approved of the final manuscript.

\section{Competing interests}

339 The authors have no financial or non-financial competing interests. 
Table 1. Antimicrobial resistance (AMR) determinants in 25 Burkholderia pseudomallei strains with verified AMR phenotypes, plus strains conferring unusual antimicrobial susceptibility and stepwise AMR variants $\$$.

\begin{tabular}{|c|c|c|c|c|c|c|c|}
\hline Patient ID & Isolate & ST & $\begin{array}{l}\text { Genome } \\
\text { accession }\end{array}$ & $\begin{array}{c}\text { Antibiotic MIC } \\
(\mu \mathrm{g} / \mathrm{mL})^{¥}\end{array}$ & $\begin{array}{c}\text { Stepwise } \\
\text { variant }^{\dagger}\end{array}$ & AMR determinant & Reference/s \\
\hline Thai patient & $316 c$ & 17 & SRR2975745 & CAZr (64) & --- & PenA $_{\text {P173S }}$ & {$[20,21]$} \\
\hline \multirow[b]{2}{*}{ Thai patient } & \multirow[b]{2}{*}{$354 e$} & \multirow[b]{2}{*}{78} & \multirow[b]{2}{*}{ AHJD00000000.1 } & CAZi (6) & --- & penA -78G $>A$ & \multirow[b]{2}{*}[7]{} \\
\hline & & & & SXTi (3) & $\begin{array}{c}\text { BpeT structural } \\
\text { variant }^{\ddagger}\end{array}$ & $\operatorname{Ptr} 1_{\mathrm{R} 21 \mathrm{fs}}$ & \\
\hline \multirow{4}{*}{$\begin{array}{c}\text { Australian } \\
\text { patient }\end{array}$} & \multirow{4}{*}{ Bp1651 } & \multirow{4}{*}{880} & \multirow{4}{*}{ SRR2102060 } & CAZr $(\geq 128)$ & penA -78G >A & PenA $_{D 245 G}$ & \multirow{4}{*}[28]{} \\
\hline & & & & $\begin{array}{l}\text { Unusual sensitivity to } \\
\text { aminoglycosides } \\
\text { (GEN, KAN) and } \\
\text { macrolides (AZM) }\end{array}$ & --- & $\mathrm{AmrB}_{\mathrm{A} 254 \mathrm{fs}}$ & \\
\hline & & & & $\operatorname{DOXr}(16)$ & --- & BPSL3085 ${ }_{\text {A88fs }}$ & \\
\hline & & & & AMCr (64/32) & penA-78G>A & PenA $_{S 78 F}$ & \\
\hline \multirow{2}{*}{ Pre-DPMS 89} & \multirow{2}{*}{ MSHR0052 } & \multirow{2}{*}{722} & \multirow{2}{*}{ SRR5818275 } & MEMr (8) & --- & $\mathrm{AmrR}_{\mathrm{E} 190^{*}}$ & \multirow{2}{*}[6]{} \\
\hline & & & & DOXr (48) & $\mathrm{AmrR}_{\mathrm{E} 190^{*}}$ & BPSL3085 $v_{211 M}$ & \\
\hline $\begin{array}{l}\text { Australian } \\
\text { patient }\end{array}$ & MSHR0292 & 236 & SRR4254580 & DOXr (16) & $\mathrm{AmrR}_{\mathrm{S} 174 \mathrm{P}}$ & BPSL3085 $\sqrt{V 40 A}$ & [26] \\
\hline \multirow{3}{*}{ P215 } & MSHR0663 & 36 & SRR2887062 & $\operatorname{SXTr}(\geq 32)$ & $\mathrm{BpeT}_{\mathrm{H} 278 \mathrm{Y}}$ & $\operatorname{Ptr}_{\text {R21fs }}$ & \multirow{3}{*}{ [6]; This study } \\
\hline & \multirow[t]{2}{*}{ MSHR0937 } & \multirow[t]{2}{*}{36} & \multirow[t]{2}{*}{ SRR2886988 } & $\begin{array}{c}\text { AMCi (12/6), MEMr } \\
(6)\end{array}$ & --- & $\mathrm{BpeR}_{\mathrm{D} 176 \mathrm{~A}}$ & \\
\hline & & & & SXTi (3) & $\mathrm{BpeR}_{\mathrm{D} 176 \mathrm{~A}}$ & MetF $_{\mathrm{Q} 142^{\star}}$ & \\
\hline \multirow{2}{*}{ P179 } & MSHR0678 & 114 & SRR6075118 & $\operatorname{MEMr}(3)$ & --- & $\mathrm{AmrR}_{\mathrm{E} 21 \mathrm{D}}$ & \multirow[b]{2}{*}[6]{} \\
\hline & MSHR0800 & 114 & SRR6075115 & MEMr (6), DOXi (8) & --- & BpeR $_{\mathrm{L} 85 f \mathrm{~s}}$ & \\
\hline \multirow{3}{*}{ P337 } & MSHR1226 & 333 & SRR9598635 & CAZr $(\geq 256)$ & --- & PenA $A_{C 75 Y}$ & [20] \\
\hline & \multirow{2}{*}{ MSHR1300 } & \multirow{2}{*}{333} & \multirow{2}{*}{ SRR6075114 } & CAZr $(\geq 256)$ & penA -78G $>A$ & PenA $_{\mathrm{C} 75 \mathrm{Y}}$ & {$[20]$} \\
\hline & & & & MEMr (4) & --- & $A m r R_{K 13 f s}$ & [6] \\
\hline
\end{tabular}




\begin{tabular}{|c|c|c|c|c|c|c|c|}
\hline & & & & SXTi (3) & --- & $\mathrm{AmrR}_{\mathrm{K} 13 \mathrm{fs}}$ & This study \\
\hline P595 & MSHR3683 & 144 & SRR11678542 & DOXi (12) & --- & BPSL3085 A88fs & {$[9,28]$} \\
\hline \multirow{2}{*}{ P608 } & \multirow{2}{*}{ MSHR4083 } & \multirow{2}{*}{36} & \multirow{2}{*}{ SRR2887030 } & SXTr (24) & $\mathrm{AmrR}_{\triangle \mathrm{A} 153-\mathrm{D} 156}$ & Dut $_{\text {G91A }}$ & \multirow{2}{*}[6]{} \\
\hline & & & & $\operatorname{MEMr}(6)$ & --- & $\mathrm{AmrR}_{\triangle \mathrm{A} 153-\mathrm{D} 156}$ & \\
\hline \multirow{3}{*}{ CF6 } & \multirow{3}{*}{ MSHR5654 } & \multirow{3}{*}{1040} & \multirow{3}{*}{ SRR3404570 } & CAZr $(\geq 256)$ & PenA 30x CNV & PenA $_{\mathrm{C} 75 \mathrm{Y}}$ & \multirow{3}{*}{ [9] } \\
\hline & & & & $\operatorname{CIPr}(\geq 32)$ & --- & GyrA $_{Y 77 S}$ & \\
\hline & & & & $\mathrm{SXTr}(\geq 32)$ & BpeT $_{\text {T314fs }}$ & Ptr1 $1_{\mathrm{R} 21 \mathrm{fs}}$ & \\
\hline \multirow{9}{*}{ CF9 } & \multirow[t]{2}{*}{ MSHR5665 } & \multirow[t]{2}{*}{252} & \multirow[t]{2}{*}{ SRR3404582 } & $\operatorname{SXTr}(\geq 32)$ & $\begin{array}{l}\operatorname{Ptr}_{1}{ }_{\mathrm{W} 116 \mathrm{R}} \\
\text { MetF }_{\mathrm{Q} 28 \mathrm{P}}\end{array}$ & Dut $_{v 77 \mathrm{~A}}$ & \multirow{9}{*}[9,23]{} \\
\hline & & & & DOXi (6) & --- & BPSL3085 A88fs & \\
\hline & \multirow[b]{2}{*}{ MSHR5666 } & \multirow[b]{2}{*}{252} & \multirow[b]{2}{*}{ SRR3404597 } & DOXs $(1)^{\#}$ & --- & BPSL3085 ${ }_{\text {A88fs }}$ & \\
\hline & & & & $\operatorname{SXTr}(\geq 32)$ & $\begin{array}{l}\text { Ptr1 }_{\mathrm{W} 116 \mathrm{R}} \\
\text { MetF }_{\mathrm{Q} 28 \mathrm{P}}\end{array}$ & Dut $_{v 77 \mathrm{~A}}$ & \\
\hline & \multirow{3}{*}{ MSHR5667 } & \multirow{3}{*}{252} & \multirow{3}{*}{ SRR3404598 } & $\operatorname{SXTr}(\geq 32)$ & $\begin{array}{l}\operatorname{MetF}_{\mathrm{N} 162 T} ; \\
\text { AmrR }_{\mathrm{L} 132 \mathrm{P}}\end{array}$ & Dut $_{\text {N99s }}$ & \\
\hline & & & & MEMr (4) & --- & $\mathrm{AmrR}_{\mathrm{L} 132 \mathrm{P}}$ & \\
\hline & & & & DOXr (48) & $\mathrm{AmrR}_{\mathrm{L} 132 \mathrm{P}}$ & BPSL3085 ${ }_{\mathrm{A} 88 \mathrm{fs}}$ & \\
\hline & \multirow[b]{2}{*}{ MSHR5669 } & \multirow[b]{2}{*}{252} & \multirow[b]{2}{*}{ SRR3404599 } & DOXi (6) & --- & BPSL3085 ${ }_{\text {A88fs }}$ & \\
\hline & & & & $\operatorname{SXTr}(\geq 32)$ & $\begin{array}{l}\text { Ptr1 }_{\text {W116R }} \\
\text { MetF }_{\text {Q28P }}\end{array}$ & Dut $_{V 77 A}$ & \\
\hline P726 & MSHR6755 & 975 & SRR6075122 & MEMr (3) & --- & $\mathrm{AmrR}_{\triangle \mathrm{V} 60-\mathrm{C} 63}$ & [6] \\
\hline \multirow{3}{*}{ P797 } & MSHR7587 & 437 & SRR6075129 & MEMr (4) & --- & $\mathrm{AmrR}_{\mathrm{G} 30 \mathrm{D}}$ & \multirow{3}{*}[6]{} \\
\hline & \multirow{2}{*}{ MSHR7929 } & \multirow{2}{*}{437} & \multirow{2}{*}{ SRR6075126 } & SXTr (4) & $\mathrm{AmrR}_{\mathrm{G} 30 \mathrm{D}}$ & $\mathrm{BPSL}_{2263_{\triangle \mathrm{D} 110-\mathrm{G} 116}}$ & \\
\hline & & & & MEMr (4) & --- & $\mathrm{AmrR}_{\mathrm{G} 30 \mathrm{D}}$ & \\
\hline \multirow{6}{*}{ CF11 } & \multirow{4}{*}{ MSHR8441 } & \multirow{4}{*}{46} & & CAZi (12) & --- & PenA 10x CNV & \\
\hline & & & & $\operatorname{SXTr}(\geq 32)$ & $\mathrm{AmrR}_{\triangle \mathrm{V} 62-\mathrm{H} 223}{ }^{\mathrm{c}}$ & Ptr1 $1_{\text {A22-G23ins_R-R-A }}$ & \\
\hline & & & SRR3382162 & $\begin{array}{l}\text { Decreased DOX } \\
\text { susceptibility (4) }\end{array}$ & $\mathrm{AmrR}_{\Delta \mathrm{V} 62-\mathrm{H} 223^{\mathrm{c}}}$ & BPSL3085 $\$ 5_{S 130 \mathrm{~L}}$ & {$\left[\begin{array}{ll}6 & 0\end{array}\right]$} \\
\hline & & & & $\begin{array}{l}\text { Decreased MEM } \\
\text { susceptibility (2) }\end{array}$ & --- & $\mathrm{AmrR}_{\Delta \mathrm{V} 62-\mathrm{H} 223^{\mathrm{c}}}$ & {$[6,9]$} \\
\hline & MSHR844? & 46 & SRR3404603 & $\operatorname{SXTr}(\geq 32)$ & $\mathrm{AmrR}_{\triangle \mathrm{V} 62-\mathrm{H} 223}{ }^{\mathrm{c}}$ & Ptr1 $1_{\text {A22-G23ins_R-R-A }}$ & \\
\hline & IVISHRర442 & 40 & SRR34040U3 & DOXi (8) & $\mathrm{AmrR}_{\Delta \mathrm{V} 62-\mathrm{H} 223^{\mathrm{C}}}$ & BPSL3085 $130 \mathrm{~L}$ & \\
\hline
\end{tabular}




\begin{tabular}{|c|c|c|c|c|c|c|c|}
\hline & & & & MEMr (3) & --- & $\mathrm{AmrR}_{\triangle \mathrm{V} 62-\mathrm{H} 223^{\mathrm{c}}}$ & \\
\hline $\begin{array}{l}\text { Non-DPMS } \\
\text { QP09 }\end{array}$ & MSHR8481 & 1378 & SRR6075123 & $\operatorname{MEMr}(6)$ & --- & $\mathrm{AmrR}_{\triangle \mathrm{A} 70-\mathrm{H} 223^{d}}$ & {$[6]$} \\
\hline P989 & $\mathrm{MSHR} 9021^{\mathrm{a}}$ & 132 & SRR6075127 & MEMr (3) & & $\mathrm{AmrR}_{\mathrm{S166 \textrm {P }}} ; \mathrm{AmrR}_{\mathrm{A} 145 \mathrm{fs}}^{\mathrm{b}}$ & [6] \\
\hline \multicolumn{8}{|c|}{ Strains with unusual antibiotic sensitivity } \\
\hline P314 & MSHR1043§ & 131 & SRR6380769 & $\begin{array}{c}\text { Unusual GEN } \\
\text { sensitivity }\end{array}$ & --- & $\mathrm{AmrA}_{\llcorner 247 \mathrm{fs}}$ & {$[14]$} \\
\hline $\begin{array}{c}\text { Malaysian } \\
\text { patient }\end{array}$ & MSHR5089§ & 881 & SRR2975737 & $\begin{array}{l}\text { Unusual sensitivity to } \\
\text { aminoglycosides } \\
\text { (GEN, KAN) and } \\
\text { macrolides (AZM) }\end{array}$ & --- & $\mathrm{AmrB}_{\text {T368R }}$ & [17] \\
\hline \multicolumn{8}{|c|}{ Strains with stepwise variants } \\
\hline P179 & MSHR0535 & 114 & SRR6075120 & DOXs $(4)^{\S}$ & BPSL3085 ${ }_{\mathrm{R} 104 \mathrm{fs}}$ & --- & [6] \\
\hline Thai patient & $354 \mathrm{e}$ & 78 & AHJD00000000.1 & AMCs (4/2) & penA -78G >A & --- & {$[7]$} \\
\hline
\end{tabular}

344 Abbreviations: AZM, azithromycin; CAZ, ceftazidime; CIP, ciprofloxacin; CNV, copy-number variation; DOX, doxycycline; GEN, gentamicin; KAN, kanamycin;

345 MEM, meropenem; MIC, minimum inhibitory concentration; SXT, co-trimoxazole

$346 \quad ¥_{i}$, intermediate; $r$, resistant; s, sensitive

347 'Where applicable

$348{ }^{\ddagger}$ Encodes an $800 \mathrm{~kb}$ inversion that direct affects $I / p E$, the intervening gene between the BpeT transcriptional regulator and its BpeEF-OprC efflux pump [7]

349 "False positive; isolate remained sensitive despite encoding a known AMR determinant. Cause of reversion unknown

350 SStrains MSHR1043 [14] and MSHR5089 [17] encode unusual antimicrobial susceptibility, and are susceptible to all five clinically-relevant antibiotics (i.e. 351 AMC, CAZ, DOX, MEM, SXT), and strains MSHR0535 and 354e encode stepwise variants that elevate the DOX and AMC MICs, respectively, but do not 352 exceed the established resistance cut-off for these antibiotics

$353{ }^{a}$ MSHR9021 was intentionally sequenced from a potentially mixed population to capture population diversity. Two AmrR mutants, AmrR $\mathrm{S}_{\mathrm{S} 166 \mathrm{P}}$ and $\mathrm{AmrR}_{\mathrm{A} 145 \mathrm{~s} s}$, 354 were present at ratios of $\sim 66 \%$ and $33 \%$, respectively [6]. In the non-mixture mode, only the dominant variant, $A m r R_{S 166 P}$, is detected.

$355{ }^{\mathrm{b}}$ Frameshift indel shortens protein length from 223 to 183 residues

$356 \quad{ }^{\mathrm{C}}$ Frameshift indel increases protein length from 223 to 285 residues

$357 \quad{ }^{\mathrm{d}}$ Frameshift indel shortens protein length from 223 to 117 residues 
bioRxiv preprint doi: https://doi.org/10.1101/720607; this version posted August 26, 2020. The copyright holder for this preprint (which was not certified by peer review) is the author/funder, who has granted bioRxiv a license to display the preprint in perpetuity. It is made available under aCC-BY-NC-ND 4.0 International license.

358 Table 2. Burkholderia pseudomallei strains phenotypically confirmed to be sensitive towards 359 the five clinically-relevant antibiotics, and associated genome data.

\begin{tabular}{|c|c|c|c|c|c|c|c|c|c|}
\hline \multirow{2}{*}{$\begin{array}{l}\text { Patient } \\
\text { ID }\end{array}$} & \multirow{2}{*}{ Isolate } & \multirow{2}{*}{ ST } & \multirow{2}{*}{$\begin{array}{c}\text { Genome } \\
\text { accession }\end{array}$} & \multicolumn{5}{|c|}{ Antibiotic MIC $(\mu \mathrm{g} / \mathrm{mL})^{*}$} & \multirow{2}{*}{ Reference/s } \\
\hline & & & & AMC & CAZ & DOX & MEM & SXT & \\
\hline $\begin{array}{l}\text { Australian } \\
\text { patient }\end{array}$ & MSHR0293 & 236 & SRR4254579 & $2 / 1$ & 1 & 1 & 0.5 & 0.4 & [26] \\
\hline \multirow{2}{*}{ P179 } & MSHR0492 & 114 & SRR6075119 & $1.5 / 0.75$ & 1.5 & 1 & 1.5 & 1 & \multirow{2}{*}{ [6] } \\
\hline & MSHR0934 & 114 & SRR6075116 & $2 / 1$ & 2 & 1 & 0.75 & 1 & \\
\hline P337 & MSHR1141 & 333 & SRR2975732 & $1.5 / 0.75$ & 1.5 & 1 & 0.75 & 0.75 & [20] \\
\hline P608 & MSHR3763 & 36 & SRR2887021 & $4 / 2$ & 2 & 0.75 & 0.75 & 3 & [6] \\
\hline P726 & MSHR5864 & 975 & SRR6075121 & $3 / 1.5$ & 1.5 & 1 & 0.75 & 1.5 & [6] \\
\hline P797 & MSHR6522 & 437 & SRR6075128 & $2 / 1$ & 1.5 & 1 & 0.5 & 1.5 & [6] \\
\hline \multirow{2}{*}{ CF1 } & MSHR0913 & 279 & SRR3404575 & $2 / 1$ & 3 & 1 & 1 & 0.5 & \multirow{2}{*}{ [9] } \\
\hline & MSHR1053 & 279 & SRR3404578 & $1.5 / 0.75$ & 2 & 1 & 0.5 & 1 & \\
\hline $\begin{array}{l}\text { Malaysian } \\
\text { patient }\end{array}$ & MSHR5093 & 881 & SRR2975738 & $6 / 3$ & 4 & 1.5 & 1 & 3 & [17] \\
\hline $\begin{array}{l}\text { Malaysian } \\
\text { patient }\end{array}$ & MSHR5104 & 881 & SRR2975740 & $2 / 1$ & 0.75 & 1 & 0.19 & 0.75 & [17] \\
\hline CF6 & MSHR5651 & 1040 & SRR3381886 & $1.5 / 0.75$ & 1.5 & 0.38 & 0.5 & 0.75 & {$[9,23]$} \\
\hline \multirow{2}{*}{ CF9 } & MSHR5662 & 252 & SRR3381885 & 2 & 2 & 2 & 0.75 & 0.5 & \multirow{2}{*}[9,23]{} \\
\hline & MSHR5670\# & 252 & SRR3404600 & $1.5 / 0.75$ & 2 & 2 & 0.75 & 0.5 & \\
\hline \multirow{2}{*}{ CF10 } & MSHR8438 & 442 & SRR3382015 & $2 / 1$ & 3 & 1 & 1 & 0.25 & \multirow{2}{*}[9,23]{} \\
\hline & MSHR8440 & 442 & SRR3404601 & $2 / 1$ & 3 & 1 & 0.75 & 0.38 & \\
\hline
\end{tabular}

360 Abbreviations: AMC, amoxicillin-clavulanate; CAZ, ceftazidime; DOX, doxycycline; MEM, meropenem;

361 ST, multilocus sequence type; SXT, co-trimoxazole 
bioRxiv preprint doi: https://doi.org/10.1101/720607; this version posted August 26, 2020. The copyright holder for this preprint (which was not certified by peer review) is the author/funder, who has granted bioRxiv a license to display the preprint in perpetuity. It is made available under aCC-BY-NC-ND 4.0 International license.

362

*According to Etesting

363 "False positive; isolate remained sensitive despite encoding known AMR determinants towards DOX

364 (BPSL3085 $\left.{ }_{\mathrm{A} 88 \mathrm{~s}}\right)$ and SXT (Ptr1 ${ }_{\mathrm{W} 116 \mathrm{R}} ;$ MetF $_{\mathrm{Q} 28 \mathrm{P}} ;$ Dutv77A $)$. Cause of reversion unknown 
bioRxiv preprint doi: https://doi.org/10.1101/720607; this version posted August 26,2020 . The copyright holder for this preprint (which was not certified by peer review) is the author/funder, who has granted bioRxiv a license to display the preprint in perpetuity. It is made available under aCC-BY-NC-ND 4.0 International license.

Table 3. ARDaP prediction in three meropenem-resistant Burkholderia pseudomallei isolates with previously unknown antimicrobial resistance (AMR) determinants.

\begin{tabular}{cccc}
\hline Strain ID & $\begin{array}{c}\text { ARDaP-predicted AMR } \\
\text { determinant }\end{array}$ & $\begin{array}{c}\text { MEM MIC } \\
(\mu \mathrm{g} / \mathrm{mL})\end{array}$ & Reference/s \\
\hline MSHR1058 & AmrR $_{\triangle \mathrm{P} 81-\mathrm{H} 2233^{\mathrm{a}}}$ & $12(\mathrm{MEM})$ & [6]; This study \\
\hline MSHR1174 & AmrR $149 \mathrm{fs}^{\mathrm{b}}$ & $6(\mathrm{MEM})$ & This study \\
\hline MSHR8777 & AmrR $_{\triangle \mathrm{A} 128-\mathrm{H} 223^{c}}$ & $4(\mathrm{MEM})$ & This study
\end{tabular}

367 'aPreviously undescribed 3' amrR deletion shortens protein length from 223 to 130 residues.

$368{ }^{b}$ Previously undescribed $11 \mathrm{bp}$ insertion shortens protein length from 223 to 178 residues.

369 'Previously undescribed 3' amrR insertion increases protein length from 223 to 621 residues. 
bioRxiv preprint doi: https://doi.org/10.1101/720607; this version posted August 26,2020 . The copyright holder for this preprint (which was not certified by peer review) is the author/funder, who has granted bioRxiv a license to display the preprint in perpetuity. It is made available under aCC-BY-NC-ND 4.0 International license.

Table 4. ARDaP detection limits for AMR determinants in synthetic mixtures.

\begin{tabular}{|c|c|c|c|}
\hline AMR determinant & $\begin{array}{l}\text { K96243 } \\
\text { gene ID }\end{array}$ & Mutation type & $\begin{array}{l}\text { AMR minor allele } \\
\text { detection (\%) }\end{array}$ \\
\hline \multicolumn{4}{|c|}{$\begin{array}{l}\text { Mixture \#1: MSHR0913 (sensitive strain) and MSHR0937 (resistant to MEM; intermediate } \\
\text { resistance to AMC and SXT) }\end{array}$} \\
\hline $\mathrm{Bpe}_{\text {Asp176Ala }}$ & BPSL0812 & Missense SNP & 20 \\
\hline MetF $_{\mathrm{Gln} 142^{*}}$ & BPSL3288 & Nonsense SNP & 25 \\
\hline \multicolumn{4}{|c|}{$\begin{array}{l}\text { Mixture \#2: MSHR0913 (sensitive strain) and MSHR8441 (resistant to MEM and SXT; intermediate } \\
\text { resistance to CAZ and DOX) }\end{array}$} \\
\hline $\mathrm{AmrR}_{\triangle \mathrm{V} 62-\mathrm{H} 223}$ & BPSL1805 & Gene loss/truncation & $30^{\dagger}$ \\
\hline BPSL3085 ${ }_{S 130 L}$ & BPSL3085 & Missense SNP & 10 \\
\hline Ptr $1_{\text {A22-G23ins_R-R-A }}$ & BPSS0039 & In-frame insertion & 25 \\
\hline penA 10x & BPSS0946 & $\begin{array}{l}\text { Copy-number variation }(10 x \\
\text { duplication) }\end{array}$ & 5 \\
\hline
\end{tabular}

371 AMR, antimicrobial resistance; CAZ, ceftazidime; CIP, ciprofloxacin; DOX, doxycycline; MEM,

372 meropenem; ND, not detected; SNP, single-nucleotide polymorphism; SXT, co-trimoxazole

$373{ }^{\dagger}$ Represents the lowest allele frequency that was consistently detected in our mixed strain dataset. 


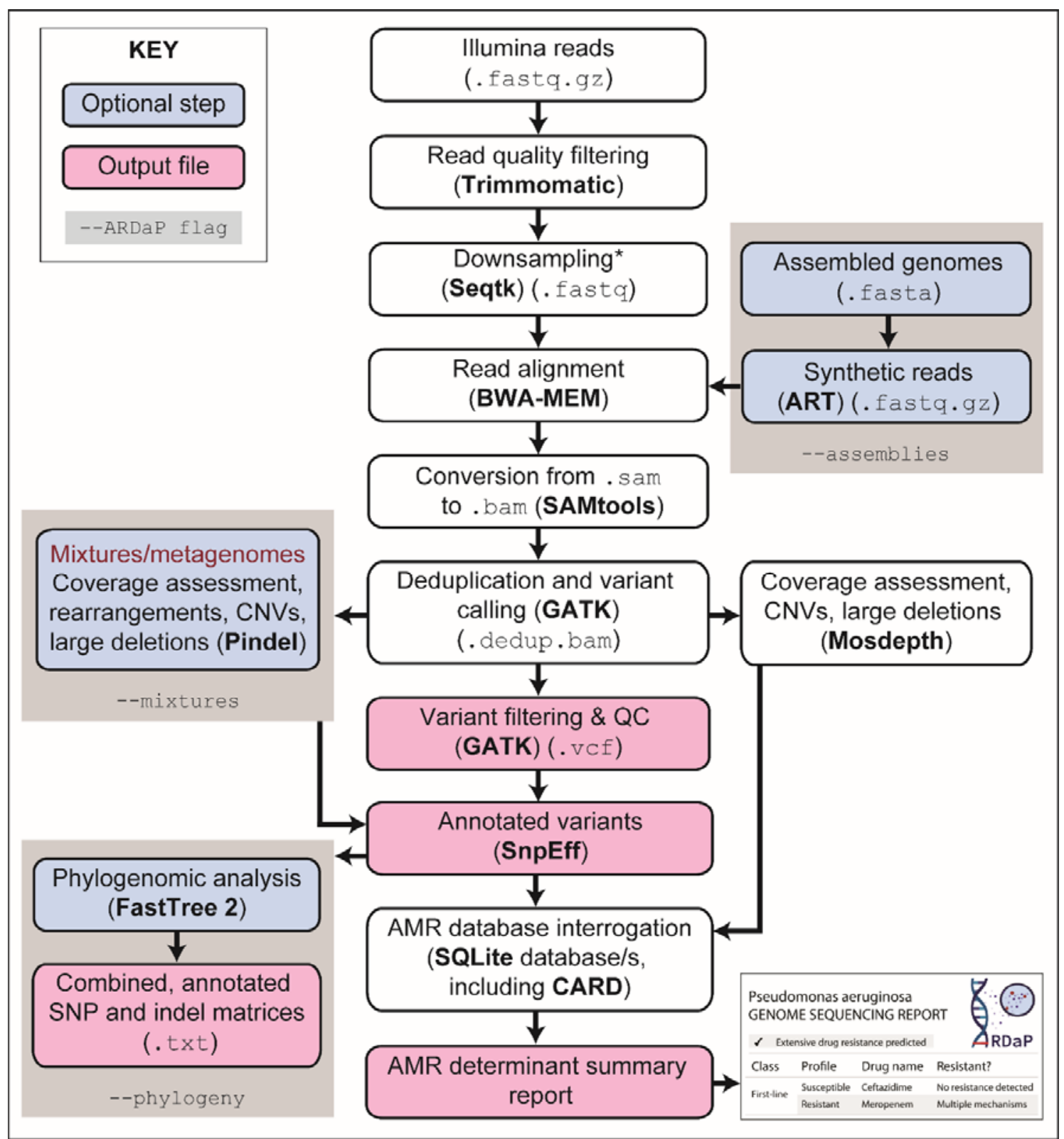

377 Figure 1. ARDaP pipeline. The user inputs assembled genome/s or raw sequencing reads, and a

378 reference genome sequence. ARDaP then performs read alignment, read processing, deduplication,

379 and variant identification. An optional phylogenetic analysis is also performed (if specified). Coverage

380 assessment is undertaken on either single or mixed genomes (if specified); genetic variants are then annotated and antimicrobial resistance database/s interrogated. Finally, ARDaP produces a summary report of antimicrobial resistance determinants for each strain (Figure 2). ${ }^{*}$ Downsampling is carried out by default but can be turned off using the --size 0 flag in ARDaP. 
bioRxiv preprint doi: https://doi.org/10.1101/720607; this version posted August 26, 2020. The copyright holder for this preprint (which was not certified by peer review) is the author/funder, who has granted bioRxiv a license to display the preprint in perpetuity. It is made available under aCC-BY-NC-ND 4.0 International license.

\section{BURKHOLDERIA PSEUDOMALLEI \\ GENOME SEQUENCING REPORT \\ NOT FOR DIAGNOSTIC USE}

\begin{tabular}{|l|l|l|l|}
\hline Patient Name & James Smith & Barcode & BARCODE \\
\hline Birth Date & $1 / 01 / 1990$ & Patient ID & MSHR5654 \\
\hline Location & Darwin & Sample Type & Blood \\
\hline Sample Source & Blood & Sample Date & $2020-07-30$ \\
\hline Sample ID & MSHR5654 & Sequenced From & Cultured isolate \\
\hline Reporting Lab & RDH & Report Date $/$ ime & 2020-07-30 \\
\hline Requested By & Dr. Requestor Name & Requestor Contact & req_contact@genome.com \\
\hline
\end{tabular}

Summary
The specimen was interogated with the Burkholderia pseudomallei database

It is predicted to be resistant to Ceftazidime, Trimethoprim/sulfamethoxazole, Ciprofloxacin.

\begin{tabular}{|c|c|c|c|c|}
\hline \multicolumn{5}{|c|}{ Drug Susceptibility } \\
\hline \multirow{4}{*}{\multicolumn{2}{|c|}{$\begin{array}{l}\text { Resistance is reported when a high-confidence resistance conferring mutation is detected. "No } \\
\text { mutation detected" does not exclude the possibility of resistance }\end{array}$}} & $\square$ & \multicolumn{2}{|c|}{ No drug resistance predicted } \\
\hline & & $\square$ & \multicolumn{2}{|c|}{ Mono-resistance predicted } \\
\hline & & $\square$ & \multicolumn{2}{|c|}{ Multi-drug resistance predicted } \\
\hline & & $\nabla$ & \multicolumn{2}{|c|}{ Extensive drug resistance predicted } \\
\hline Drug class & Interpretation & Drug & & Resistance determinant \\
\hline \multirow{2}{*}{ First-line } & Sensitive & \multicolumn{2}{|c|}{ Meropenem } & No resistance detected \\
\hline & Resistant & \multicolumn{2}{|c|}{ Ceftazidime } & Multiple determinants \\
\hline \multirow{4}{*}{ Second-line } & Sensitive & \multicolumn{2}{|c|}{ Imipenem } & No resistance detected \\
\hline & Resistant & \multicolumn{2}{|c|}{ Trimethoprim/sulfamethoxazole } & Multiple determinants \\
\hline & Sensitive & \multicolumn{2}{|c|}{ Amoxicillin/Clavulanic acid } & No resistance detected \\
\hline & Sensitive & \multicolumn{2}{|c|}{ Doxycycline } & No resistance detected \\
\hline \multicolumn{5}{|c|}{ Extended/non-clinical drug susceptibility } \\
\hline Drug class & Interpretation & \multicolumn{2}{|c|}{ Drug } & Resistance determinant \\
\hline Tertiary & Resistant & \multicolumn{2}{|c|}{ Ciprofloxacin } & gyrA BPSL2521 Tyr 77Ser \\
\hline \multicolumn{5}{|c|}{ Intrinsic drug resistance/unusual drug susceptibility } \\
\hline Drug class & Interpretation & \multicolumn{2}{|c|}{ Drug } & Mechanism of sensitivity \\
\hline Intrinsic & Resistant & \multicolumn{2}{|c|}{ Gentamicin } & No sensitivity detected \\
\hline
\end{tabular}

384

Antimicrobial determinant details

gyrA|BPSL2521|Tyr $77 \operatorname{Ser} \mid$ CIPr|Confers CIP MIC $532 \mu \mathrm{g} / \mathrm{mL}$

penA|BPSS0946|Cys75Tyr CAZr Confers CAZ MIC $\$ 256 \mu \mathrm{g} / \mathrm{mL}$; also known as PenA Cys69Tyr

BPSS0039 ptr1|Arg21fs $\mid$ SXTr $\mid<$ PRECURSOR MUTATION $>$. Confers SXT MIC $\$ 32 \mu \mathrm{g} / \mathrm{mL}$ in combination with BpeT Thr314fs, BpeT His $274 \mathrm{Tyr}$, or BpeT loss. Confers intermediate resistance by itself BPSS0290|bpeT|Thr314fs $\mid$ SXTr $\mid<$ PRECURSOR MUTATION $>$. Confers SXT MIC $532 \mu \mathrm{g} / \mathrm{mL}$ in combination with Ptr 1 Arg20fs

penA BPSS0946 upregulation|CAZr|0|10x copy number confers MIC=12 ug/uL; 2x copy number does not increase MIC (Viberg et al., 2017 MBio 8(2):00356-17)

BPSS0039|ptr1 | loss $\mid$ SXTr 1 |Confers SXT resistance in combination with efflux pump upregulation. Confers intermediate resistance by itself

BPSS0290|bpeT $\mid$ loss $\mid$ SXTr| $|0|<$ PRECURSOR MUTATION>. Isolates with this mutation are more likely to develop SXT resistance

\section{Natural variation that does not confer antimicrobial resistance}

amrR|BPSL1805|Val222f $\mathrm{f}_{\mathbf{s}}$ None|Known variation in amrR that does not cause antibiotic resistance

385 Figure 2. Example antimicrobial resistance (AMR) summary report produced by ARDaP. The

386 final step in the ARDaP pipeline is the production of a user-friendly report that summarises patient and

387 sample details, confirms that the given isolate is the expected species (in this case, Burkholderia

388 pseudomalleI), identifies any predicted AMR (including what mutation/s has/have been detected) and

389 what antibiotic/s have been affected, identifies unusual antimicrobial sensitivity and natural variation

390 that does not confer AMR, and identifies stepwise mutations that lower the barrier to AMR

391 development. 


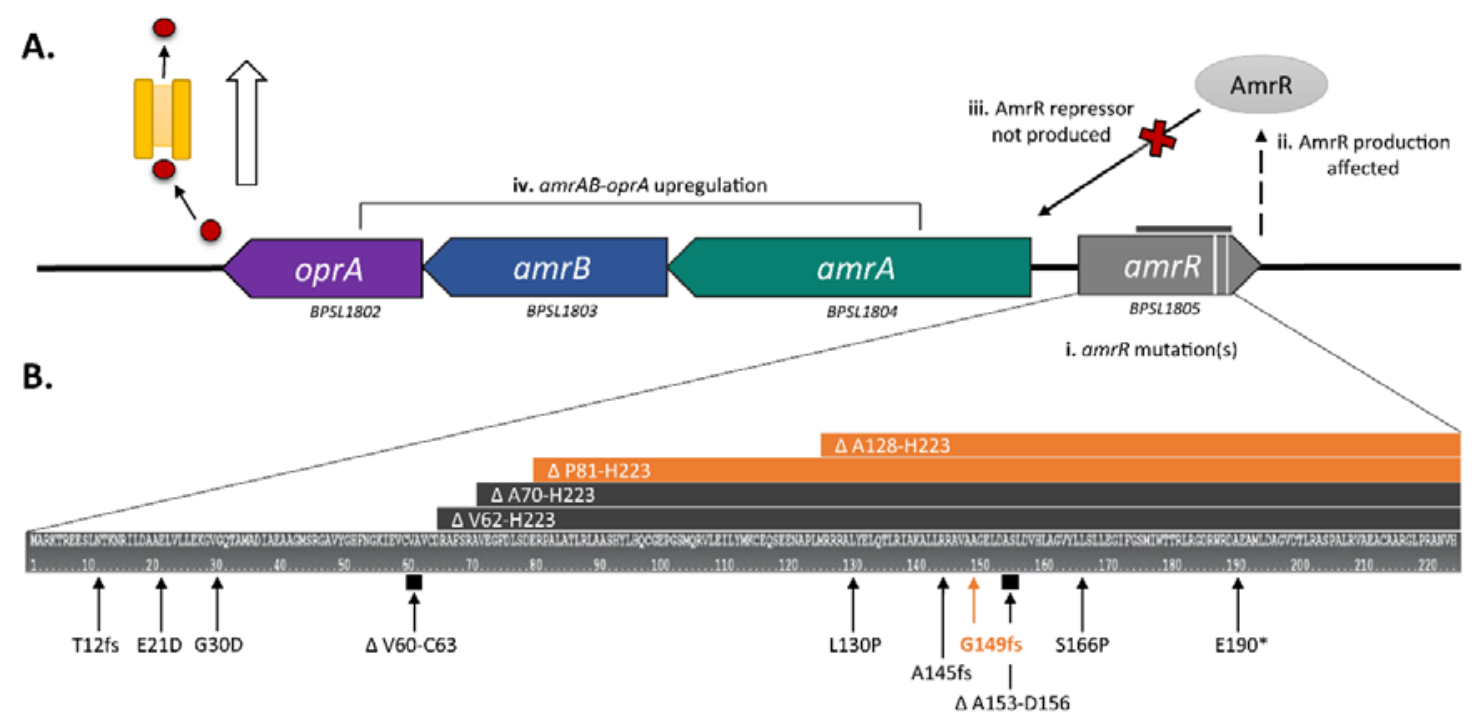

Figure 3. Operon organisation of the Burkholderia pseudomallei AmrAB-oprA resistance-

394 nodulation-division efflux pump and loss-of-function mutations in its TetR-type regulator,

395 AmrR. A. Transcriptional organisation of the amrR (BPSL1805), amrA (BPSL1804), amrB

396 (BPSL1803) and oprA (BPSL1802) operon, and summary of how (i) amrR mutations cause (ii) loss-of-

397 function of AmrR, which (iii) no longer represses expression of the resistance-nodulation-division

398 AmrAB-OprA efflux pump, resulting in (iv) efflux pump over-expression and resistance to meropenem

399 and aminoglycoside antibiotics. B. Distribution and annotation of amrR mutations. Eleven previously

400 observed amrR mutations (in black) [6] have been augmented with three novel mutations identified in

401 the current study (orange); $\mathrm{AmrR}_{\mathrm{G} 149 \mathrm{fs}}, \mathrm{AmrR}_{\triangle \mathrm{P} 81-\mathrm{H} 223}$, and $\mathrm{AmrR}_{\triangle \mathrm{A} 128-\mathrm{H} 223}$, all of which cause amrR

402 loss-of-function, resulting in efflux pump overexpression and meropenem resistance. 
404 1. Didelot X, Bowden R, Wilson DJ, Peto TEA, Crook DW. Transforming clinical

microbiology with bacterial genome sequencing. Nat Rev Genet 2012; 13(9): 601-12.

2. Lakin SM, Dean C, Noyes NR, et al. MEGARes: an antimicrobial resistance database for high throughput sequencing. Nucleic acids research 2017; 45(D1): D574-d80.

3. Gupta SK, Padmanabhan BR, Diene SM, et al. ARG-ANNOT, a new bioinformatic tool to discover antibiotic resistance genes in bacterial genomes. Antimicrobial agents and chemotherapy 2014; 58(1): 212-20.

4. Hunt M, Mather AE, Sanchez-Buso L, et al. ARIBA: rapid antimicrobial resistance genotyping directly from sequencing reads. Microbial genomics 2017; 3(10): e000131.

5. Jia B, Raphenya AR, Alcock B, et al. CARD 2017: expansion and model-centric curation of the comprehensive antibiotic resistance database. Nucleic acids research 2017; 45(D1): D566-d73.

6. Sarovich DS, Webb JR, Pitman MC, et al. Raising the stakes: Loss of efflux-pump regulation decreases meropenem susceptibility in Burkholderia pseudomallei. Clinical infectious diseases : an official publication of the Infectious Diseases Society of America 2018.

7. Hayden HS, Lim R, Brittnacher MJ, et al. Evolution of Burkholderia pseudomallei in recurrent melioidosis. PloS one 2012; 7(5): e36507.

8. Tristram S, Jacobs MR, Appelbaum PC. Antimicrobial resistance in Haemophilus influenzae. Clin Microbiol Rev 2007; 20(2): 368-89.

9. Viberg LT, Sarovich DS, Kidd TJ, et al. Within-host evolution of Burkholderia pseudomallei during chronic infection of seven Australasian cystic fibrosis patients. mBio 2017; 8(2).

10. Nicoloff H, Hjort K, Levin BR, Andersson DI. The high prevalence of antibiotic heteroresistance in pathogenic bacteria is mainly caused by gene amplification. Nat Microbiol 2019; 4(3): 504-14.

11. Schweizer HP. Mechanisms of antibiotic resistance in Burkholderia pseudomallei: implications for treatment of melioidosis. Future Microbiol 2012; 7(12): 1389-99.

12. Dance D. Treatment and prophylaxis of melioidosis. International journal of antimicrobial agents 2014; 43(4): 310-8.

13. Limmathurotsakul D, Peacock SJ. Melioidosis: A clinical overview. Br Med Bull 2011; 99(1): 125-39.

14. Price EP, Sarovich DS, Mayo M, et al. Within-host evolution of Burkholderia pseudomallei over a twelve-year chronic carriage infection. mBio 2013; 4(4): e0038813.

15. Crowe A, McMahon N, Currie BJ, Baird RW. Current antimicrobial susceptibility of first-episode melioidosis Burkholderia pseudomallei isolates from the Northern Territory, Australia. International journal of antimicrobial agents 2014; 44(2): 160-2.

16. Maloney S, Engler C, Norton R. Epidemiological cut-off value of clinical isolates of Burkholderia pseudomallei from northern Queensland to meropenem, ceftazidime, trimethoprim/sulfamethoxazole and doxycycline by the microbroth dilution method. Journal of global antimicrobial resistance 2017; 10: 291-4.

17. Podin Y, Sarovich DS, Price EP, et al. Burkholderia pseudomallei isolates from Sarawak, Malaysian Borneo, are predominantly susceptible to aminoglycosides and macrolides. Antimicrobial agents and chemotherapy 2014; 58(1): 162-6.

18. Rholl DA, Papp-Wallace KM, Tomaras AP, Vasil ML, Bonomo RA, Schweizer HP. Molecular investigations of PenA-mediated $\beta$-lactam resistance in Burkholderia pseudomallei. Front Microbiol 2011; 2: 139.

19. Sam IC, See KH, Puthucheary SD. Variations in ceftazidime and amoxicillinclavulanate susceptibilities within a clonal infection of Burkholderia pseudomallei. Journal of clinical microbiology 2009; 47(5): 1556-8. 
456 20. Sarovich DS, Price EP, Limmathurotsakul D, et al. Development of ceftazidime resistance in an acute Burkholderia pseudomallei infection. Infect Drug Resist 2012; 5: 129-32.

21. Tribuddharat C, Moore RA, Baker P, Woods DE. Burkholderia pseudomallei class A $\beta$-lactamase mutations that confer selective resistance against ceftazidime or clavulanic acid inhibition. Antimicrobial agents and chemotherapy 2003; 47(7): 20827.

22. Chirakul S, Norris MH, Pagdepanichkit S, et al. Transcriptional and posttranscriptional regulation of PenA $\beta$-lactamase in acquired Burkholderia pseudomallei $\beta$-lactam resistance. Sci Rep 2018; 8(1): 10652.

23. Price EP, Viberg LT, Kidd TJ, Bell SC, Currie BJ, Sarovich DS. Transcriptomic analysis of longitudinal Burkholderia pseudomallei infecting the cystic fibrosis lung. Microbial genomics 2018; 4(8).

24. Chantratita N, Rholl DA, Sim B, et al. Antimicrobial resistance to ceftazidime involving loss of penicillin-binding protein 3 in Burkholderia pseudomallei. Proceedings of the National Academy of Sciences of the United States of America 2011; 108(41): 1716570.

25. Podnecky NL, Rhodes KA, Mima T, et al. Mechanisms of resistance to folate pathway inhibitors in Burkholderia pseudomallei: deviation from the norm. mBio 2017; 8(5).

26. Webb JR, Price EP, Currie BJ, Sarovich DS. Loss of methyltransferase function and increased efflux activity leads to doxycycline resistance in Burkholderia pseudomallei. Antimicrobial agents and chemotherapy 2017; 61(6).

27. Rhodes KA, Somprasong N, Podnecky NL, Mima T, Chirakul S, Schweizer HP. Molecular determinants of Burkholderia pseudomallei BpeEF-OprC efflux pump expression. Microbiology (Reading, England) 2018; 164(9): 1156-67.

28. Bugrysheva JV, Sue D, Gee JE, et al. Antibiotic resistance markers in Burkholderia pseudomallei strain Bp1651 identified by genome sequence analysis. Antimicrobial agents and chemotherapy 2017; 61(6).

29. Currie BJ. Melioidosis: evolving concepts in epidemiology, pathogenesis, and treatment. Seminars in respiratory and critical care medicine 2015; 36(1): 111-25.

30. Wuthiekanun V, Amornchai P, Saiprom N, et al. Survey of antimicrobial resistance in clinical Burkholderia pseudomallei isolates over two decades in Northeast Thailand. Antimicrobial agents and chemotherapy 2011; 55(11): 5388-91. 